\title{
Flow Cytometry-Assisted Detection of Adenosine in Serum with an Immobilized Aptamer Sensor
}

\author{
Po-Jung Jimmy Huang and Juewen Liu* \\ Department of Chemistry, Waterloo Institute for Nanotechnology, University of Waterloo, \\ 200 University Avenue West, Waterloo, Ontario, N2L 3G1, Canada
}

Email address: $\underline{\text { liujw@uwaterloo.ca }}$

\section{Received 15 December 2009}

Date accepted 2 April 2010

Published online 20 April 2010

* To whom correspondence should be addressed. Phone: 519-888-4567 ext 38919. E-mail: liujw@uwaterloo.ca.

This document is the Accepted Manuscript version of a Published Work that appeared in final form in Analytical Chemistry, copyright @ American Chemical Society after peer review and technical editing by publisher. To access the final edited and published work see Huang, P.-J. J., \& Liu, J. (2010). Flow Cytometry-Assisted Detection of Adenosine in Serum with an Immobilized Aptamer Sensor. Analytical Chemistry, 82(10), 4020-4026. https://doi.org/10.1021/ac9028505 


\begin{abstract}
Aptamers are single-stranded nucleic acids that can selectively bind to essentially any molecule of choice. Because of their high stability, low cost, ease of modification and availability through selection, aptamers hold great promise in addressing key challenges in bioanalytical chemistry. In the past fifteen years many highly sensitive fluorescent aptamer sensors have been reported. However, few such sensors showed high performance in serum samples. Further challenges related to practical applications include detection in a very small sample volume and a low dependence of sensor performance on ionic strength. We report the immobilization of an aptamer sensor on a magnetic microparticle and the use of flow cytometry for detection. Flow cytometry allows the detection of individual particles in a capillary and can effectively reduce the light scattering effect of serum. Since DNA immobilization generated a highly negatively charged surface and caused an enrichment of counter ions, the sensor performance showed a lower salt dependence. The detection limits for adenosine are determined to be 178 and $167 \mu \mathrm{M}$ in buffer and in 30\% serum, respectively. Finally, we demonstrated that the detection can be carried out in $10 \mu \mathrm{L}$ of $90 \%$ human blood serum.
\end{abstract}




\section{INTRODUCTION}

One of the key challenges in bioanalytical chemistry is the detection of disease-related proteins and metabolites in biological fluids. ${ }^{1}$ For practical applications, the sample volume should be as small as possible. ${ }^{2}$ For example, glucose sensors have revolutionized the health care of diabetic patients; currently only a few micro-liters of blood is needed. ${ }^{3}$ While protein enzymes and antibodies are the traditional molecules of choice for making biosensors, aptamers are emerging as a promising alternative due to their small sizes, high stability (especially DNA aptamers), ease of modification and immobilization, and excellent target recognition properties. ${ }^{4-16}$ Aptamers are single-stranded nucleic acids that can selectively bind to target molecules. ${ }^{17-19}$ Because of their useful properties, aptamers have been widely used for constructing biosensors. Among the various signal transduction methods, fluorescence has been the most often used due to its high sensitivity and versatility. ${ }^{5,20-27}$ In addition to intensity-based detection, fluorescence wavelength shift, anisotropy, lifetime, and energy transfer have all been reported for designing aptamer-based sensors. ${ }^{5}$

Most fluorescent aptamer sensors involve cuvette-based measurements, in which the sensor and target analyte are mixed in a cuvette and the change of fluorescence signal is monitored with a fluorometer. With significant progresses being made, one of the intrinsic limitations of such cuvette-based detection is its incompatibility with serum samples. This is in part due to the cloudy optical appearance of serum; most light is absorbed or scattered, resulting in a very high background that masks fluorescence signal. For this reason, a majority of fluorescent sensors are demonstrated only in clean buffers and very few are shown to work in serum, in particular, undiluted serum. ${ }^{21,28}$ On the other hand, at least $100-500 \mu \mathrm{L}$ of sample is needed to fill a fluorescence cuvette. For practical application, it is desirable to decrease the sample volume to a level comparable to that used for glucose detection (e.g. $\leq 10 \mu \mathrm{L})$.

Even though serum samples are chemically complex, it has been demonstrated that aptamers can still selectively recognize their targets in serum. ${ }^{29-32}$ If one can separate the fluorescence detection step from 
serum samples, fluorescent aptamer sensors can still be used for serum analysis. ${ }^{33,34}$ Among the various methods for DNA separation or buffer exchange, such as electrophoresis, dialysis, immobilization, centrifugation, and chromatography, we aim to achieve separation without actually performing an additional separation step (i.e. couple separation with detection). Herein, we report the immobilization of a fluorescent aptamer sensor on magnetic microparticles (MMPs) and the use of flow cytometry for detection of adenosine and related metabolites in serum. In flow cytometry, particles are analyzed individually through a capillary, and the optical path length in serum is significantly reduced. We demonstrate that as little as $10 \mu \mathrm{L}$ serum is sufficient for each assay, and the sensor shows a similar performance in serum as in buffer. In recent years, flow cytometry has become a routine technique in many hospitals for the analysis of blood samples and therefore flow-based analytical methods should be feasible in clinical or hospital settings. While flow cytometry has been used for aptamer-based cell detection, ${ }^{35-38}$ this is the first report to use this technique for small molecule analysis.

\section{EXPERIMENTAL SECTION}

Materials. The FAM-labeled biotinylated DNA aptamer and the complementary quencher-labeled DNA were purchased from Integrated DNA Technologies Inc (Coralville, IA). The DNA sequences are: 5'-FAM-TCACTGACCTGGGGGAGTATTGCGGAGGAAGGTTTTTT-Biotin $\quad$ and 5'CCCAGGTCAGTG-Iowa Black FQ, and Iowa Black FQ is a fluorescence quencher. Streptavidincoupled $2.8 \mu \mathrm{m}$ Dynabeads M-270 were purchased from Invitrogen. Adenosine, other nucleosides, $\mathrm{NaCl}$, Tris, and HEPES buffer were purchased from Mandel Scientific (Guelph, Ontario, Canada). ATP, AMP, and cAMP were purchased from Sigma. Human blood serum was purchased from Fisher Scientific. All buffers and solutions were made with Millipore water. Nucleoside solutions were prepared to be $50 \mathrm{mM}$. To fully dissolve adenosine, cAMP, and guanosine, the solutions were heated to a boiling temperature. 
DNA Conjugation and Adenosine Detection. Before taking the MMPs out of the stock vial, the vial was vortexed to ensure that the particles were homogenously distributed. The particles were washed following the instructions provided by the vendor with $2 \times \mathrm{B} \& \mathrm{~W}$ buffer $(10 \mathrm{mM}$ Tris- $\mathrm{HCl}, \mathrm{pH} 7.5,1$ $\mathrm{mM}$ EDTA, $2 \mathrm{M} \mathrm{NaCl}$ ) to remove the preservatives and were temporary stored in $1 \mathrm{x} \mathrm{B} \& \mathrm{~W}$ buffer before use. For DNA conjugation, $3 \mu \mathrm{L}$ of $1 \mu \mathrm{M}$ the fluorescent biotinylated DNA was incubated with 6 $\mu \mathrm{L}$ of $1 \mu \mathrm{M}$ quencher-labeled DNA in $100 \mu \mathrm{L} 1 \times \mathrm{B} \& \mathrm{~W}$ buffer at $40{ }^{\circ} \mathrm{C}$ for 10 min and was slowly cooled down to room temperature. $1 \mu \mathrm{L}$ of the MMPs were then coupled with the DNA at room temperature for 20-30 min. Excess DNA was discarded with the help of a magnet and the coated MMPs were washed with a small amount of $1 x$ B\&W buffer $(30 \mu \mathrm{L} \times 2)$ and buffer A $(25 \mathrm{mM}$ HEPES/150mM $\mathrm{NaCl})(30 \mu \mathrm{L} \times 1)$. Various amount of adenosine was added to react with the aptamers functionalized MMPs in $50 \mu \mathrm{L}$ of buffer A or in serum (diluted with the same buffer from $100 \%$ serum) for $20 \mathrm{~min}$. The samples were then diluted to $300 \mu \mathrm{L}$ with buffer A for flow cytometry analysis. To prepare centrifuged serum samples, a bench top centrifuge running at $15000 \mathrm{rpm}$ for $20 \mathrm{~min}$ at $4{ }^{\circ} \mathrm{C}$ was used. The fluorescence was measured either with a BD FACSVantage SE flow cytometer (with MMPs) or a Perkin Elmer LS-55 fluorometer (without MMPs). For flow cytometry assays, normal operation conditions with phosphate buffered saline based sheath fluids were used and at least 20000 events were counted for each sample. For assays with the fluorometer, $400 \mu \mathrm{L}$ of the samples were used. The solutions were excited at $490 \mathrm{~nm}$ and fluorescence emission at $520 \mathrm{~nm}$ was collected.

NaCl-Dependent Studies and Kinetics Assays. The sensor particles were prepared as described above and were diluted with buffers containing varying concentrations of $\mathrm{NaCl}$. The fluorescence 20 min after adding $2 \mathrm{mM}$ adenosine was measured with the flow cytometer or fluorometer. For kinetics studies, $10 \mu \mathrm{L}$ of the sensor MMPs were used and the particles were finally dispersed in $100 \mu \mathrm{L}$ buffer A. Adenosine was added to a final concentration of $2 \mathrm{mM}$ or $10 \mathrm{mM}$. At designated time points, $10 \mu \mathrm{L}$ of MMPs were taken out and diluted to $300 \mu \mathrm{L}$ with buffer A for flow cytometry analysis. 
$\xi$-Potential Measurement. The $\xi$-potential was measured with a Malvern Zetasizer nano ZS instrument with a dip-cell. $1 \mu \mathrm{L}$ of the DNA conjugated MMPs were dispersed in $1 \mathrm{~mL}$ buffer A and the measurement was performed at $25^{\circ} \mathrm{C}$ with ten measurement circles.

\section{RESULTS AND DISCUSSION}

Sensor Design. To demonstrate the concept, we chose to use a well-characterized fluorescent aptamer sensor shown in Figure 1A. ${ }^{20,25,39}$ It contains an adenosine aptamer DNA labeled with a 5'-FAM and a 3'-biotin. This aptamer can bind ATP, AMP, and adenosine, ${ }^{40}$ and has been widely used as a model system for designing aptamer-based sensors. The aptamer portion is colored in green and two short DNA spacers were added (in black and gray) between the aptamer and the two labels. After hybridization with a 12-mer DNA labeled with a 3'-quencher, the FAM emission is suppressed. Addition of adenosine (denoted as a red star in the figure) induces a structure switch in the aptamer to allow target binding, resulting in the release of the quencher-labeled DNA and an increased fluorescence signal. $^{20,25}$ As shown in Figure 1C, this sensor can produce a 20-fold fluorescence enhancement in the presence of $10 \mathrm{mM}$ adenosine in buffer A (25mM HEPES, pH 7.6, 150mM NaCl). However the fluorescence was largely obscured in a human blood serum sample (30\% serum) with the same concentration of the sensor and adenosine (Figure 1D). The fluorescence enhancement was less than 1fold in this case due to the strong light scattering effect of serum, which represents a $>20$-fold decrease in the signal-to-background ratio. If $80 \%$ serum was used, fluorescence enhancement can barely be observed (see Supporting Information). 

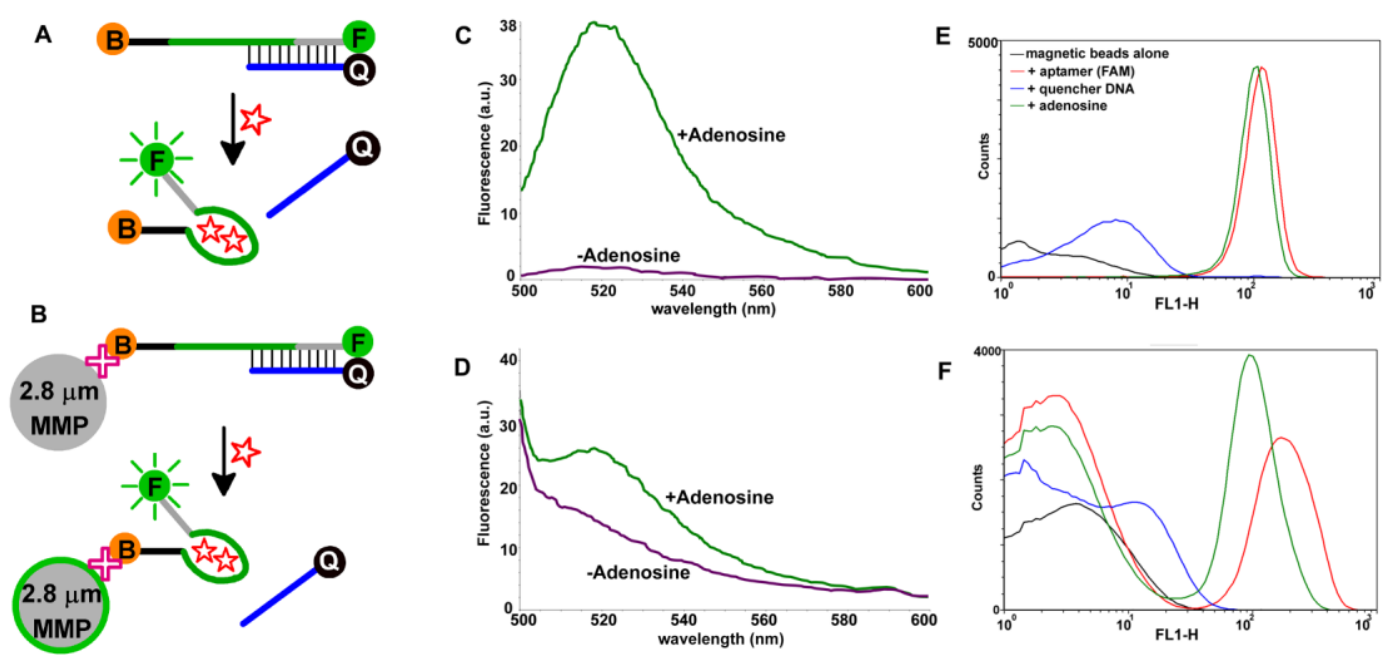

Figure 1. Non-immobilized (A) and immobilized (B) fluorescent aptamer sensor for adenosine based on the displacement of a quencher-labeled DNA by adenosine (adenosine is denoted as a red star). F = FAM fluorophore and $\mathrm{Q}=$ quencher. Fluorescence spectra of the non-immobilized sensor in buffer (C) and in 30\% serum (D). Flow cytometry histograms of the immobilized sensor in buffer (E) and in $30 \%$ serum $(\mathrm{F})$.

To minimize serum-induced optical background, this sensor was immobilized on a streptavidin-coated MMP $($ diameter $=2.8 \mu \mathrm{m})$ through the biotin label as shown in Figure 1B. Five thymine nucleotides were introduced as a spacer between the biotin and the aptamer to minimize possible effects of surface immobilization on aptamer binding. Due to the very high binding affinity between biotin and streptavidin, a simple mixing and incubation is sufficient for immobilization. As specified by the vendor, on average $1.8 \times 10^{6}$ single-stranded DNAs can be immobilized on the surface of each particle, although no information on the DNA length was given. We estimated that $1.2 \times 10^{6}$ DNAs were attached to each particle (DNA length $=38$ mer, see Supporting Information), which is comparable with the vendor's value. Free DNAs in the supernatant were removed with the help of a magnet. Release of the quencher-labeled strand by the target molecule renders the MMP highly fluorescent and can be detected by flow cytometry. 
Under an optical microscope, the aptamer-functionalized MMPs were fully dispersed and no aggregates were observed, which makes flow cytometry analysis uncomplicated. A representative flow cytometry histogram of the immobilized adenosine sensor in buffer A is shown in Figure 1E. The $x$-axis is the FAM fluorescence intensity and the $y$-axis is the number of particles. Under experimental conditions, unmodified MMPs have an average fluorescence of $\sim 2$ (Figure 1E, the black curve). After adding the FAM-labeled biotinylated DNA, the peak shifted to $\sim 110$ (red curve). With the quencherlabeled DNA, the fluorescence shifted back to $\sim 8$ (blue curve), which corresponds to a 14-fold fluorescence decrease. This indicates that a high DNA hybridization efficiency was maintained on the particle surface. After incubating with $10 \mathrm{mM}$ adenosine at room temperature for $20 \mathrm{~min}$, the fluorescence signal was almost fully recovered (green curve), suggesting that adenosine can induce the structure switch of the immobilized aptamer and release the quencher-labeled DNA. A similar degree of fluorescence enhancement was also observed in serum samples as shown in Figure 1F (comparing the blue and green curves), suggesting that the effect of serum on fluorescence detection was reduced through the use of flow cytometry. The nozzle diameter of the flow cytometer is $70 \mu \mathrm{m}$, which represents a $>50$-fold reduction of light path length in serum compared to a $4 \mathrm{~mm}$ micro-cuvette. Dilution of serum by the sheath fluid should have minimal effects on the observed background reduction, because both the light path length in serum and the serum concentration contribute to light scattering, and the product of these two parameters should be a constant according to Beer's law (assuming serum only diffuses laterally). We also noticed that the histogram was broadened in the presence of serum, although the peak intensity stayed roughly the same (e.g. the red peaks with fluorescence of $\sim 100$ in Figure 1E and F). This broadening was attributed to the increased viscosity of the flow sample, ${ }^{41}$ which decreased particle flow rate in serum to produce a broader peak in the histogram. $^{42}$

The major difference between Figure $1 \mathrm{~F}$ and $1 \mathrm{E}$ is that the population of "particles" with very low fluorescence (e.g. fluorescence value $<10$ ) was significantly increased in the serum samples, possibly 
due to serum protein aggregates and cell debris that may have a similar size as the MMPs but are nonfluorescent. To quantitatively understand the effect of serum, the light scattering of the particles and its correlation with fluorescence intensity were studied. In buffer, FAM-labeled MMPs in the forward/side scatter histogram are concentrated in a small region (Figure 2A), suggesting that these MMPs are monodispersed. The position of this population was used to define a gate (the red circle) for subsequent analysis. Because of the FAM label, a population with fluorescence intensity of $\sim 100$ was observed in the fluorescence/side scatter plot (Figure 2B), and in the fluorescence histogram (Figure 2C). Figure 2DF show the same plots for serum (100\% serum, no MMPs), and a significant number of serum particles were in the gate, which were also counted as "particles". These particles, however, were non-fluorescent (Figure 2E, F). When the fluorescent MMPs were mixed with serum, the resulting histograms were essentially a simple addition of the two individual components (Figure 2G-I). Even though there are significantly more serum particles than MMPs under experimental conditions (within the gate serum:MMP = 29:1), these two populations can be clearly separated based on fluorescence.
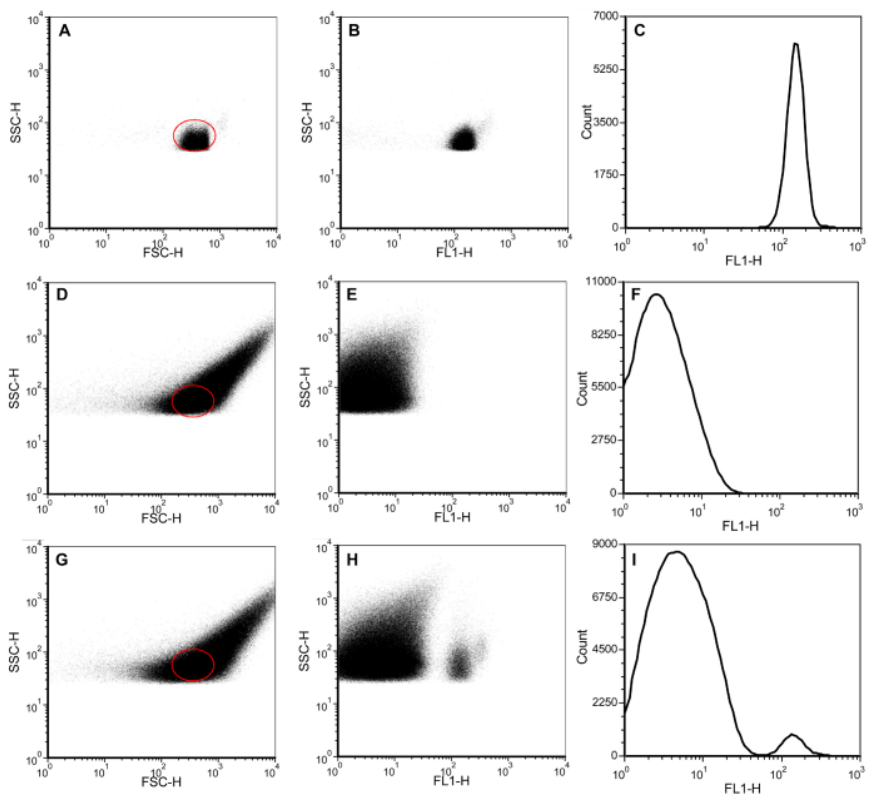

Figure 2. Histograms of forward (FSC)/side (SSC) scatter (A, D, G), fluorescence/side scatter (B, E, H), and fluorescence $(\mathrm{C}, \mathrm{F}, \mathrm{I})$ of fluorescent MMPs in buffer (A-C), pure serum (D-F), and fluorescent MMPs in serum (G-I). 
NaCl-Dependent Studies. To optimize the detection conditions, systematic studies on the $\mathrm{NaCl}-$ dependent behavior of both the non-immobilized and immobilized sensor were performed in buffer. The buffer contained $25 \mathrm{mM}$ HEPES, pH 7.6 with varying concentrations of $\mathrm{NaCl}$ up to $1 \mathrm{M}$ (Figure 3). For the non-immobilized sensor, the assays were performed in a cuvette with a fluorometer. There is a clear trend of signal inhibition at high salt concentrations (Figure 3A, open squares). In the presence of $1 \mathrm{M}$ $\mathrm{NaCl}$, the signal decreased by $\sim 70 \%$ compared to that in $25 \mathrm{mM} \mathrm{NaCl}$. The adenosine sensing process can be considered to be a competition between the aptamer binding to adenosine and to the quencherlabeled DNA (Figure 1A). It is known that double-stranded DNAs are more stable in the presence of higher salt while the aptamer complex stability may be less affected by salt because not every nucleotide in the aptamer is paired. ${ }^{43,44}$ This can explain the suppressed signal enhancement for the nonimmobilized sensor. Interestingly, we observed a much less pronounced salt-dependent response for the immobilized sensor analyzed by flow cytometry (Figure 3B). In the range of 25 to $300 \mathrm{mM} \mathrm{NaCl}$, the average fluorescence stayed at $\sim 50$ after reacting with $2 \mathrm{mM}$ adenosine. The background signal in the absence of adenosine was also more stable across the $1 \mathrm{M}$ salt range tested (solid circles). The ability to generate less salt-dependent signals is very important, because the ionic strengths are sometimes unknown for practical samples.
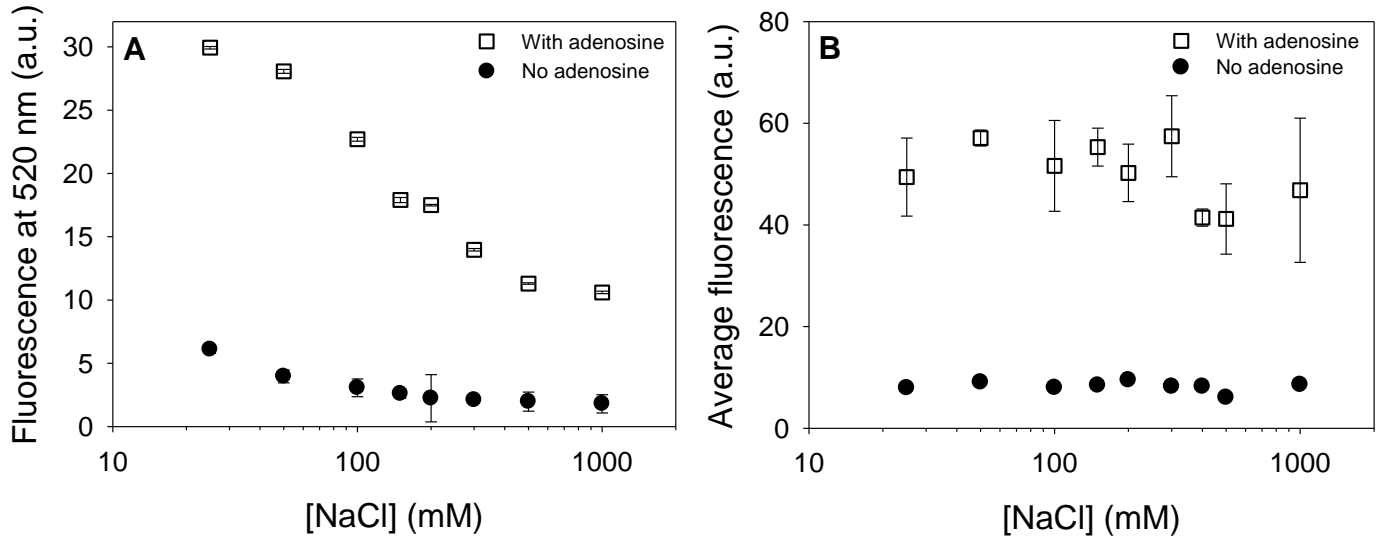

Figure 3. The fluorescence signal of the non-immobilized (A) and immobilized sensor (B) with increasing $\mathrm{NaCl}$ concentrations. In both plots, the sensor signals before and 20 min after adding $2 \mathrm{mM}$ adenosine were compared. 
DNA is a polyanion and each phosphate carries a negative charge under physiological pH. DNA immobilization creates a densely packed and highly negatively charged layer on the microparticle surface. Based on the particle size and DNA density, it is estimated that there is one DNA every $20 \mathrm{~nm}^{2}$, and each DNA carries 50 negative charges at $\mathrm{pH}$ 7.6. A direct consequence of a highly negatively charged surface is the enrichment of counterions (in this case, $\mathrm{Na}^{+}$), and the enrichment factor compared to the bulk concentration is given by the Boltzmann equation $\exp \left(|\phi e| / k_{B} T\right)$, where $\phi$ is the surface potential, $e$ is the elemental charge, $k_{B}$ is the Boltzmann constant, and $T$ is temperature in Kelvin. The measured $\zeta$ potential with $25 \mathrm{mM} \mathrm{NaCl}$ at $\mathrm{pH} 7.6$ was $-22 \mathrm{mV}$. This, however, was the potential at the plane of shear and the true surface potential should be higher. If $\zeta$ potential is used as the surface potential for a conserved estimate, an enrichment factor of 2.2 is obtained for $25 \mathrm{mM} \mathrm{NaCl}$ to give a local $\left[\mathrm{Na}^{+}\right]$of $55 \mathrm{mM}$. Because the enrichment factor increases exponentially with the surface potential, the actual enrichment should be much higher. On the other hand, the surface potential decreases with increasing salt concentration (see Supporting Information). Therefore, the counterion enrichment factor should decrease in high salt. The overall effect is a higher enrichment factor in low salt and a lower enrichment in high salt. This may explain the salt independent behavior for the immobilized sensor. The drastically increased local counterion concentration around DNA-functionalized gold nanoparticles has also been suggested to be responsible for protecting DNA from nuclease degradation by Mirkin and coworkers. $^{45}$

Reaction Kinetics. To understand the time-dependent signal change of the immobilized sensor, kinetic studies have been performed by taking aliquots out and analyzing by flow cytometry at designated time points after adding 2 or $10 \mathrm{mM}$ adenosine. As can be observed in Figure 4, most of fluorescence change occurred in the first $5 \mathrm{~min}$ and the signals reached saturation in about $20 \mathrm{~min}$ for both samples. Therefore, we chose to use a $20 \mathrm{~min}$ reaction time for the immobilized sensor for most studies in this work. A higher adenosine concentration produced a higher final signal, which formed the basis for quantitative analysis. The fact that each sample can quickly reach a stable final signal suggests 
that the immobilized system can reach equilibrium, just like the non-immobilized sensor, ${ }^{20}$ and the released quencher-labeled DNA can still re-hybridize with the immobilized aptamer DNA. Otherwise, the fluorescence signal in the $2 \mathrm{mM}$ adenosine sample should continue to increase until it reached the same final value as in the $10 \mathrm{mM}$ one.

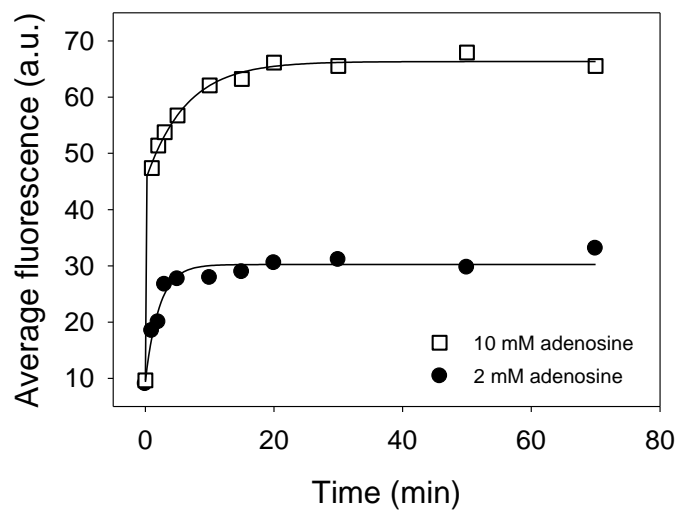

Figure 4. Kinetics of fluorescence enhancement for the immobilized sensor in the presence of 2 and 10 mM adenosine analyzed with flow cytometry.

Sensitivity and Selectivity. With the optimized detection conditions, the sensitivity and selectivity of the sensor was examined in buffer A with a 20 min reaction time. Figure 5A shows the flow cytometry histograms of the sensor in the presence of varying concentrations of adenosine, and a gradual shift in the peak fluorescence can be observed. If the average fluorescence in Figure 5A is plotted against adenosine concentration (Figure 5B), an overall sigmoid shaped curve is obtained with a slow signal increase at low target concentrations followed by a sharp rise at high concentrations. The low concentration region can be fit to a linear response (inset) with a detection limit of $178 \mu \mathrm{M}$ adenosine based on the $3 \sigma /$ slope calculation. The selectivity of the sensor was also tested with $10 \mathrm{mM}$ of various nucleosides. As can be seen from Figure 5C, with $10 \mathrm{mM}$ uridine, cytodine, or guanosine, the average fluorescence stayed at the same position as the untreated sensor, suggesting that the high selectivity of the original aptamer was maintained. The response of the sensor to several analogs of adenosine, including ATP, AMP, and cAMP was also tested (Figure 5D), and the sensor is also sensitive to these compounds but with slightly lower responses, which is consistent with literature reports. ${ }^{20,40}$ The 
sensitivity of the sensor is high enough for detecting some of these molecules under physiological conditions (e.g. $>2 \mathrm{mM}$ for ATP). ${ }^{46}$ To distinguish these analogs individually, however, aptamers with better selectivity are needed. ${ }^{47}$
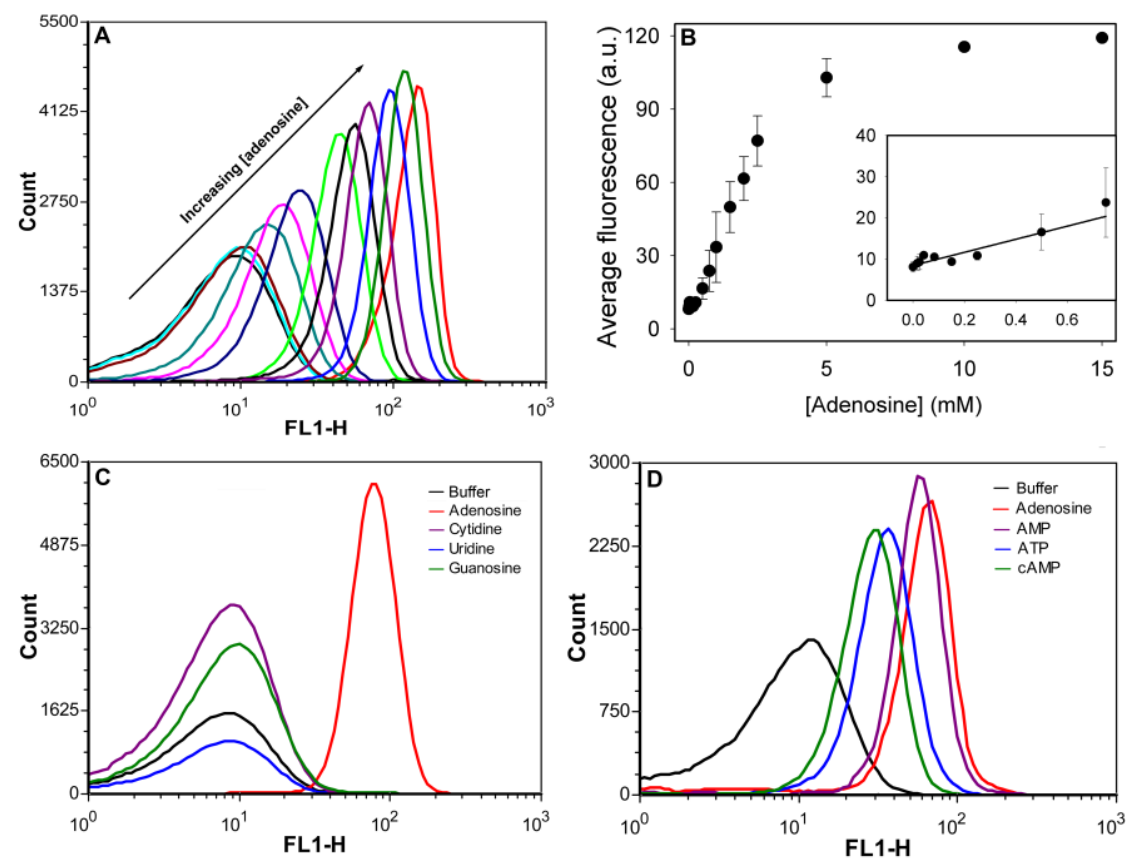

Figure 5. Flow cytometry histograms of the sensor in the presence of varying concentration of adenosine (A). Sensitivity (B) and selectivity (C, D) of the immobilized adenosine sensor in buffer. In (C and D), $10 \mathrm{mM}$ adenosine, adenosine analogs or other nucleosides were used.

Detection in Serum. One of the main motivations of this study is to test whether the immobilized aptamer sensor can be used for detection in blood serum. We have shown in Figure 1F that this sensor indeed works in serum. For quantitative detection, samples with $30 \%$ serum were spiked with varying concentrations of adenosine and other procedures were the same as in the case of detection in clean buffers. The fluorescence signal increased with increasing adenosine and a detection limit of $167 \mu \mathrm{M}$ was obtained (Figure 6A), which is similar to the detection limit in buffer A. The immobilized sensor also showed a very high selectivity in serum (Figure 6B), and only adenosine induced a fluorescence enhancement. These results suggest that serum did not change the molecular recognition property of the sensor. ${ }^{29,48}$ 
We next challenged the sensor with $90 \%$ serum. Pure serum was not tested because $10 \%$ of the volume was used to add adenosine. As can be observed in Figure 6C, even though very high serum peaks were present in these samples, $200 \mu \mathrm{M}$ adenosine can still be detected (inset). If the serum samples were centrifuged (with adenosine already dissolved) to remove aggregated proteins and cell debris before reacting with the sensor, the number of residual serum particles was comparable to that of MMPs (Figure 6D), and centrifugation did not change the sensitivity of the sensor.
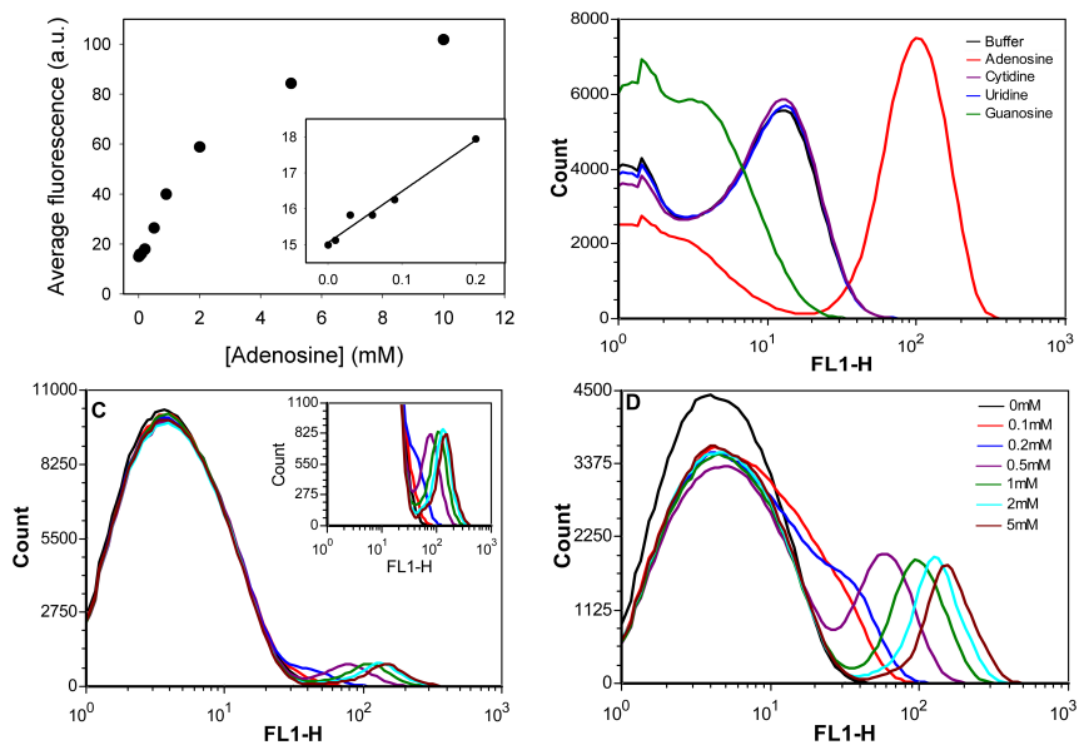

Figure 6. Sensitivity (A) and selectivity (B) of the immobilized adenosine sensor in $30 \%$ serum. Flow cytometry histograms of the sensor response to varying concentrations of adenosine in $90 \%$ serum (C) and in $90 \%$ serum after centrifugation (D) to remove aggregated proteins in serum. (C) and (D) share the figure legend, and the inset of (C) highlights the MMP population.

In all the experiments reported so far, the sensor particles were dispersed in $>50 \mu \mathrm{L}$ of samples. Because the sensor was immobilized, it is possible to perform detection in a very small volume. To demonstrate this, the sensor was reacted in $10 \mu \mathrm{L}$ of $90 \%$ serum with varying concentrations of adenosine. Because a volume of $10 \mu \mathrm{L}$ was insufficient for flow cytometry-based detection, a dilution with buffer A to $300 \mu \mathrm{L}$ was performed right before the flow cytometry analysis. Although the 
adenosine concentration was diluted by 30 -fold, a detection limit of $\sim 200 \mu \mathrm{M}$ was achieved for both the non-centrifuged (Figure 7A) and centrifuged (Figure 7B) serum. Therefore, this method can be applied for the analysis of very tiny volume of samples. With such a low volume of serum and centrifugation to remove large particles in serum, the population from serum is only $5 \%$ of the MMPs. The fact that the sensor was immobilized represents another advantage of this approach. Although the volume was increased, the fluorescence signal was not diluted because of immobilization.
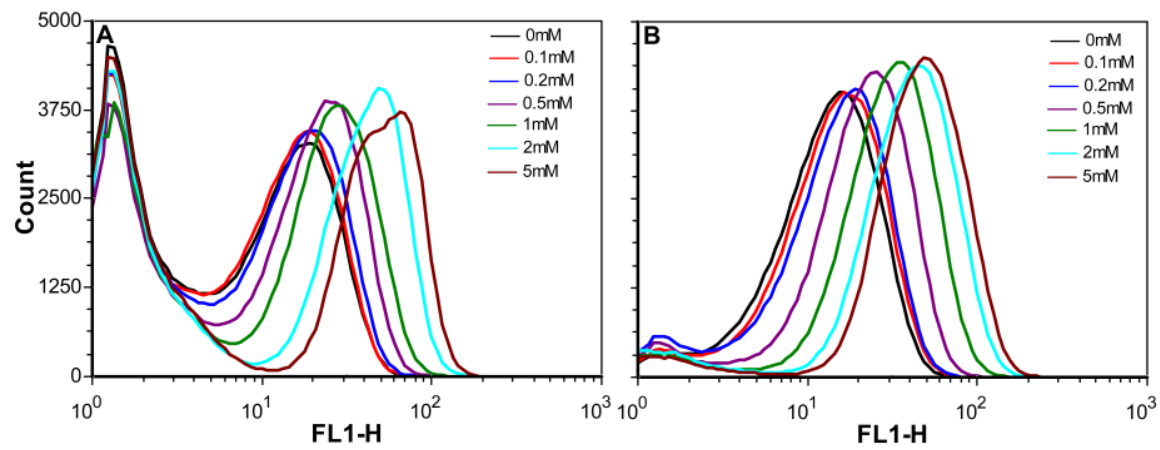

Figure 7. Detection of adenosine in $10 \mu \mathrm{L}$ of serum samples for the non-centrifuged (A) and centrifuged (B) serum.

Advantage of Sensor Immobilization. There are several important features for this new detection method. First, based on the knowledge that aptamers can still selectively bind their targets in serum, flow cytometry provides a convenient means for separating fluorescence detection from the serum sample matrix. By applying a gate on the sensor particles, a large fraction of particles are outside the gate and are not considered for analysis. For the serum originated events within the gate, they are almost non-fluorescent and can be distinguished from the sensor particles in the presence of adenosine. Therefore, fluorescent aptamer sensors can be used for serum analysis through a simple immobilization step. Second, the amount of DNA used for detection can be significantly reduced. For a typical experiment in a cuvette (e.g. $400 \mu \mathrm{L}$ of 50 nM DNA), 20 pmol DNA is needed for each assay. For the immobilized sensor, if 10,000 particles are counted and each particle contains $1.2 \times 10^{6}$ DNAs, only 17 fmol DNA is needed. This represents a $>1000$-fold reduction in DNA usage. Even if the number of 
particles is increased by 10 -fold to allow a faster detection, the save in DNA is still greater than 100fold. Third, the immobilized sensor shows a lower dependence on the sample ionic strength. Fourth, immobilization allows the use of as low as $10 \mu \mathrm{L}$ of sample. Finally, flow cytometry is known for its multiplex capability. By using different aptamers, fluorophores and particle sizes, multiple targets can potentially be detected in one-pot with a very small volume of blood sample.

\section{CONCLUSIONS}

In conclusion, we have developed a new aptamer-based assay with flow cytometry. This method takes advantage of the unique capability of flow cytometry in detecting particles individually in a capillary and thus drastically decreasing the light path length in serum. The sensor particles showed a comparable performance in blood serum as in clean buffers. We also observed a lower salt dependence of the immobilized sensor, which was attributed to the enrichment of couterions on the particle surface by the densely immobilized DNA. The method allows sensitive and selective detection in a small volume of concentrated human blood serum, and it should be applicable to other fluorescent sensors through immobilization.

\section{ACKNOWLEDGMENT.}

We are grateful for the help of Mihaela Savulescu for flow cytometry analysis, Professor Tong Leung for experiments performed with the fluorometer, and Professor $\mathrm{Pu}$ Chen for the $\xi$-potential measurement. We thank the University Of Waterloo for financial support.

\section{SUPPORTING INFORMATION AVAILABLE}

Quantification of DNA on particle surface, flow cytometry histograms of the sensor in buffer and in serum, and discussion about surface potential and enrichment of counterions. This material is available free of charge via the Internet at http://pubs.acs.org. 


\section{REFERENCES}

(1) Fernie, A. R.; Trethewey, R. N.; Krotzky, A. J.; Willmitzer, L. Nat. Rev. Mol. Cell Biol. 2004, 5, 763-769.

(2) Gygi, S. P.; Rist, B.; Gerber, S. A.; Turecek, F.; Gelb, M. H.; Aebersold, R. Nat. Biotechnol. 1999, 17, 994-999.

(3) Heller, A.; Feldman, B. Chem. Rev. 2008, 108, 2482-2505.

(4) Cho, E. J.; Lee, J.-W.; Ellington, A. D. Annu. Rev. Anal. Chem. 2009, 2, 241-264.

(5) Liu, J.; Cao, Z.; Lu, Y. Chem. Rev. 2009, 109, 1948-1998.

(6) Zhao, W.; Brook, M. A.; Li, Y. Chembiochem 2008, 9, 2363-2371.

(7) Song, S. P.; Wang, L. H.; Li, J.; Zhao, J. L.; Fan, C. H. Trac-Trends in Anal. Chem. 2008, 27, 108-117.

(8) Mok, W.; Li, Y. Sensors 2008, 8, 7050-7084.

(9) Navani, N. K.; Li, Y. Curr. Opin. Chem. Biol. 2006, 10, 272-281.

(10) Famulok, M.; Hartig, J. S.; Mayer, G. Chem. Rev. 2007, 107, 3715-3743.

(11) Breaker, R. R. Nature 2004, 432, 838.

(12) Sefah, K.; Phillips, J. A.; Xiong, X.; Meng, L.; Van Simaeys, D.; Chen, H.; Martin, J.; Tan, W. Analyst 2009, 134, 1765-1775.

(13) Lin, C.; Katilius, E.; Liu, Y.; Zhang, J.; Yan, H. Angew. Chem., Int. Ed. 2006, 45, 5296-5301.

(14) Huang, C.-C.; Chiu, S.-H.; Huang, Y.-F.; Chang, H.-T. Anal. Chem. 2007, 79, 4798-4804.

(15) Huang, Y. C.; Ge, B. X.; Sen, D.; Yu, H. Z. J. Am. Chem. Soc. 2008, 130, 8023-8029.

(16) Wang, D. Y.; Lai, B. H. Y.; Sen, D. J. Mol. Biol. 2002, 318, 33-43.

(17) Ellington, A. D.; Szostak, J. W. Nature 1990, 346, 818-822.

(18) Tuerk, C.; Gold, L. Science 1990, 249, 505-510.

(19) Joyce, G. F. Ann. Rev. Biochem. 2004, 73, 791-836.

(20) Nutiu, R.; Li, Y. J. Am. Chem. Soc. 2003, 125, 4771-4778. 
(21) Yang, C. J.; Jockusch, S.; Vicens, M.; Turro, N. J.; Tan, W. Proc. Natl. Acad. Sci. U.S.A. 2005, $102,17278-17283$.

(22) Tang, Z. W.; Mallikaratchy, P.; Yang, R. H.; Kim, Y. M.; Zhu, Z.; Wang, H.; Tan, W. H. J. Am. Chem. Soc. 2008, 130, 11268-11269.

(23) Jhaveri, S.; Rajendran, M.; Ellington, A. D. Nat. Biotechnol. 2000, 18, 1293-1297.

(24) Jhaveri, S. D.; Kirby, R.; Conrad, R.; Maglott, E. J.; Bowser, M.; Kennedy, R. T.; Glick, G.; Ellington, A. D. J. Am. Chem. Soc. 2000, 122, 2469-2473.

(25) Nutiu, R.; Li, Y. Chem. Eur. J 2004, 10, 1868-1876.

(26) Hafner, M.; Schmitz, A.; Grune, I.; Srivatsan, S. G.; Paul, B.; Kolanus, W.; Quast, T.; Kremmer, E.; Bauer, I.; Famulok, M. Nature 2006, 444, 941-944.

(27) Elbaz, J.; Shlyahovsky, B.; Li, D.; Willner, I. Chembiochem 2008, 9, 232-239.

(28) Stojanovic, M. N.; de Prada, P.; Landry, D. W. J. Am. Chem. Soc. 2001, 123, 4928-4931.

(29) Zuo, X.; Xiao, Y.; Plaxco, K. W. J. Am. Chem. Soc. 2009, 131, 6944-6945.

(30) Baker, B. R.; Lai, R. Y.; Wood, M. S.; Doctor, E. H.; Heeger, A. J.; Plaxco, K. W. J. Am. Chem. Soc. 2006, 128, 3138.

(31) Liu, J.; Mazumdar, D.; Lu, Y. Angew. Chem., Int. Ed. 2006, 45, 7955-7959.

(32) Yigit, M. V.; Mazumdar, D.; Kim, H.-K.; Lee, J. H.; Odintsov, B.; Lu, Y. ChemBioChem 2007, $8,1675-1678$.

(33) Wang, Y.; Liu, B. Langmuir 2009, 25, 12787-12793.

(34) Song, M.; Zhang, Y.; Li, T.; Wang, Z.; Yin, J.; Wang, H. J. Chromatogr. A 2009, 1216, 873-878.

(35) Herr, J. K.; Smith, J. E.; Medley, C. D.; Shangguan, D.; Tan, W. Anal. Chem. 2006, 78, 29182924.

(36) Xu, Y.; Phillips, J. A.; Yan, J. L.; Li, Q. G.; Fan, Z. H.; Tan, W. H. Anal. Chem. 2009, 81, 74367442.

(37) Cao, X. X.; Li, S. H.; Chen, L. C.; Ding, H. M.; Xu, H.; Huang, Y. P.; Li, J.; Liu, N. L.; Cao, W. H.; Zhu, Y. J.; Shen, B. F.; Shao, N. S. Nucleic Acids Res. 2009, 37, 4621-4628. 
(38) Raddatz, M. S. L.; Dolf, A.; Endl, E.; Knolle, P.; Famulok, M.; Mayer, G. Angew. Chem., Int. Ed. 2008, 47, 5190-5193.

(39) Nutiu, R.; Li, Y. Angew. Chem., Int. Ed. 2005, 44, 1061-1065.

(40) Huizenga, D. E.; Szostak, J. W. Biochemistry 1995, 34, 656-665.

(41) Storie, I.; Sawle, A.; Goodfellow, K.; Whitby, L.; Granger, V.; Reilly, J. T.; Barnett, D. Cytometry Part B-Clinical Cytometry 2003, 55B, 1-7.

(42) Schiro, P. G.; Kuyper, C. L.; Chiu, D. T. Electrophoresis 2007, 28, 2430-2438.

(43) Schildkr.C; Lifson, S. Biopolymers 1965, 3, 195-\&.

(44) Bishop, G. R.; Ren, J. S.; Polander, B. C.; Jeanfreau, B. D.; Trent, J. O.; Chaires, J. B. Biophys. Chem. 2007, 126, 165-175.

(45) Seferos, D. S.; Prigodich, A. E.; Giljohann, D. A.; Patel, P. C.; Mirkin, C. A. Nano Lett. 2009, 9, $308-311$.

(46) Zheng, D.; Seferos, D. S.; Giljohann, D. A.; Patel, P. C.; Mirkin, C. A. Nano Lett. 2009, 9, 32583261.

(47) Sazani, P. L.; Larralde, R.; Szostak, J. W. J. Am. Chem. Soc. 2004, 126, 8370-8371.

(48) Swensen, J. S.; Xiao, Y.; Ferguson, B. S.; Lubin, A. A.; Lai, R. Y.; Heeger, A. J.; Plaxco, K. W.; Soh, H. T. J. Am. Chem. Soc. 2009, 131, 4262-4266. 